\title{
An introductory guide to quality improvement in perioperative medicine
}

\author{
Yusuke Mazda ${ }^{1} \cdot$ Kazuyoshi Aoyama ${ }^{2,3}$
}

Received: 2 January 2021 / Accepted: 22 February 2021 / Published online: 4 March 2021

(c) Japanese Society of Anesthesiologists 2021

Keywords Model for improvement · Plan-Do-Study-Act cycle · Quality improvement · SQUIRE guidelines

\section{What is QI?}

In the Japanese healthcare system, QI often means "Quality Indicator," which refers to measuring the quality of healthcare quantitatively with numbers or statistics, such as how many patients fall during hospitalization or how often surgical site infection occurs. However, in general, QI refers to "Quality Improvement" and encompasses a larger concept of improvement in healthcare. In this context, QI in healthcare can be defined as a systematic framework to improve patient care with long-term, continuous, and sustainable changes [1, 2]. From here on, we will refer to QI as Quality Improvement, and it is worth noting that its history in healthcare spans over at least the past 100 years [3]. The Donabedian model was the initial framework for healthcare quality, which includes elements of structure, process, and outcomes. This model laid the foundation for the Institute for Healthcare Improvement's (IHI) Model for Improvement with three types of measures (outcome, process, and balancing) [4]. Currently, QI is considered a fundamental part of modern medicine in many countries, for physicians and is even taught to medical students [5]. In many healthcare systems, we recognize there can be a significant gap between what we learn from clinical research and what we do in our daily practices [6]. It is quite common to encounter

Kazuyoshi Aoyama

kazu.aoyama@utoronto.ca

1 Division of Obstetric Anesthesia, Department of Anesthesiology, Saitama Medical Center, Saitama Medical University, Kawagoe, Japan

2 Department of Anesthesia and Pain Medicine, Hospital for Sick Children, University of Toronto, 555 University Ave, \#2211, Toronto, ON M5G 1X8, Canada

3 Program in Child Health Evaluative Sciences, SickKids Research Institute, Toronto, ON, Canada situations where we cannot adapt the results from randomized controlled trials and systematic reviews, or even apply guidelines as intended to achieve the best outcomes for the patients we see and treat. Evidence-based medicine (EBM) focuses on "doing the right things" and pursuing the best medicine, while QI focuses more on "doing things right" and determining how to implement changes [7]. These concepts do not conflict, rather complement each other. They are inseparable for developing better clinical outcomes, although QI has different scientific approaches than clinical trials (Table 1).

Running a QI project may sound difficult, but it simply requires understanding and following a general, structured flow. First, one must clarify the clinical goal, which is motivated by providing better patient care in the institute. QI's target in healthcare is well known as the "Triple Aim" which refers to improving patient outcomes, enhancing the patient experience, and reducing per capita healthcare cost. One of these three aims is set as a primary outcome of the QI project. Second, one must specify the project aim in the form of an Aim Statement using SMART criteria (SMART is an acronym meaning Specific, Measurable, Achievable, Realistic, and Timely). Of note, one must be careful not to rush to a solution as it is important to take the time to characterize the causes of a target issue or understand the relevant processes in the local context. After developing the aim, a multidisciplinary dedicated team is organized to conduct a route cause analysis for detecting specific causes of the target issue from diverse perspectives. The team presumes the barriers or obstacles for the improvement and discusses which items can be improved in the institute by considering available human resources, financial circumstances, and stakeholders' engagements. Generally speaking, a successful QI project is supported by multiple departments, involving chiefs in clinical service and hospital executives. The team decides which data to collect for the outcome, process, and 


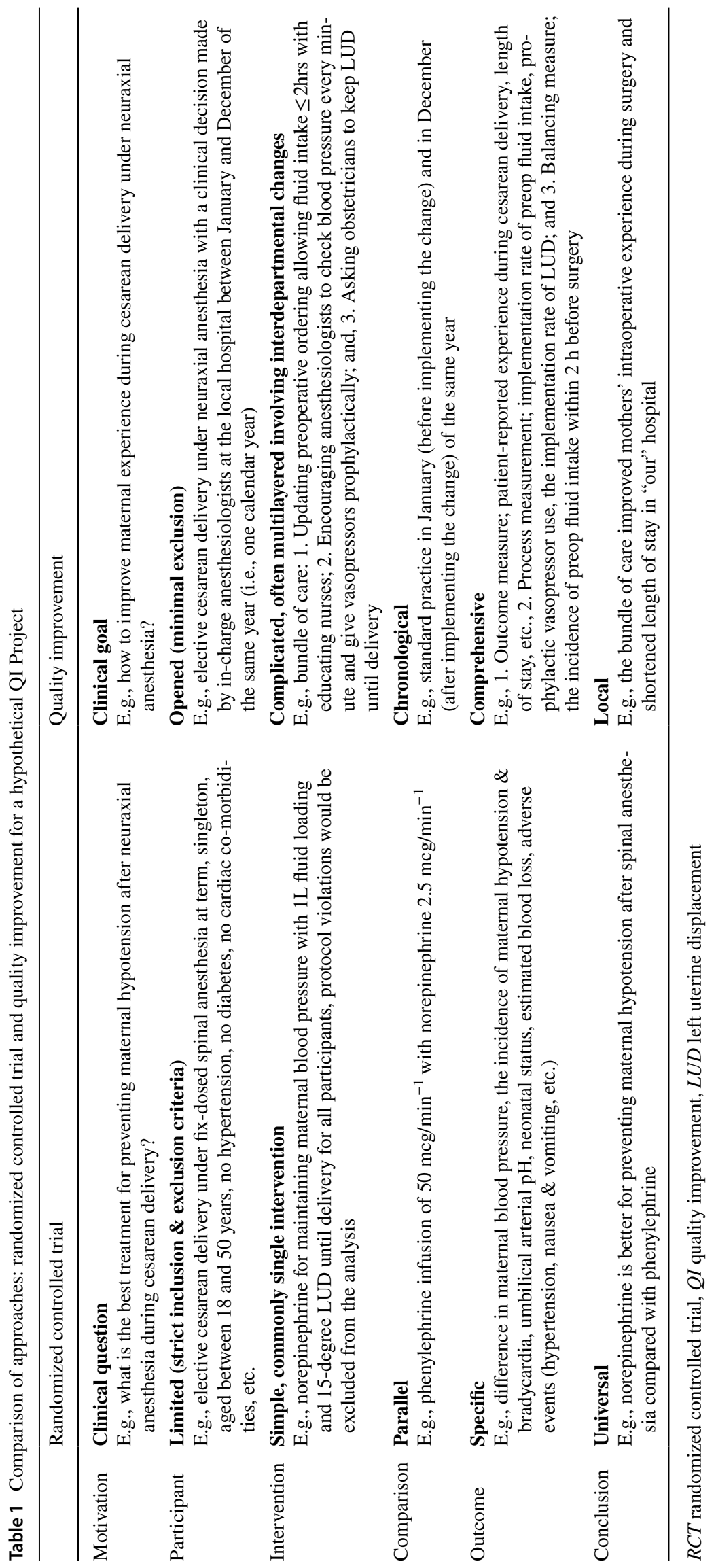


balancing measurements to provide the evidence that will show how clinical outcomes change due to the improvement. To achieve continuous QI, the project should be monitored using improvement tools, such as the Plan-Do-Study-Act (PDSA) cycle, and Six Sigma and Lean strategies [8]. The PDSA cycle is the most commonly applied tool for QI due to its simplicity and feasibility. This model provides a framework of improvement for establishing, examining, and implementing changes; Plan - think out what we should change; Do-test the change; Study - learn from the results of the change; and, Act-upgrade the regular protocol (Fig. 1). We have created a project example to demonstrate using the PDSA cycle for continuous QI.

In our hypothetical QI example, an obstetric anesthesia team receives negative feedback from patients about uncomfortableness related to an elective cesarean delivery. The

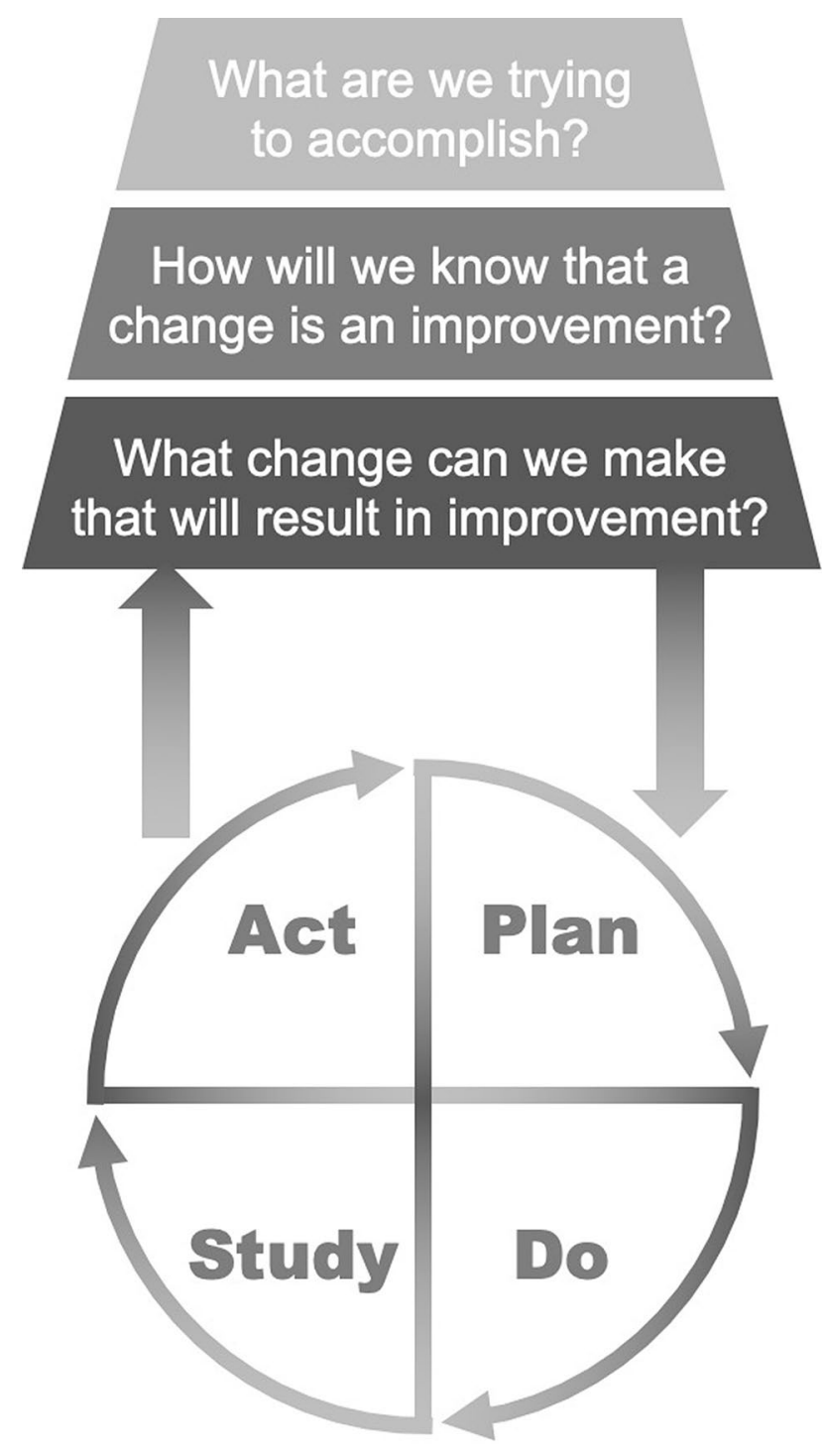

Fig. 1 The model for improvement team finds that preoperative thirst is the leading cause of the patients' complaints, so they attempt to shorten the period of NPO order for clear fluid before surgery. As obstetricians routinely order the NPO, the anesthesia team thinks that if anesthesiologists make the order, patients will drink clear fluid as much as possible before surgery (Plan). The team picks a week for testing the new preoperative NPO elective order (Do), followed by analyzing the number of NPO violations. The analysis reveals that only $5 \%$ of the patients take clear fluid within $2 \mathrm{~h}$ before surgery (Study), and the team decides to implement the new NPO order to upgrade their daily practice (Act). The PDSA cycle is then repeated on the change to their practice to measure if an improvement is observed. Most QI projects require several PDSA cycles, and the team adjusts the protocol until they observe the improvement. The improvement can be visualized using a Shewhart control chart (Fig. 2).

\section{Perioperative quality improvement: what anesthesiologists can contribute?}

Like other specialties, anesthesia has been developing QI in perioperative medicine, and Chahal et al. recently published their elegant QI project in Anaesthesia [9]. They conducted a five-year QI project with four-staged implementations in Melbourne, Australia, and achieved a significant reduction in perioperative venous thromboembolism (VTE) (from 3.1 per 1,000 to 0.6 per 1,000 ). The hospital realized that the generally applied 'one-size-fits-all' approach did not address patient-specific risk and considered clinical balance between VTE prophylaxis and bleeding. After a baseline audit, they developed the surgical-thrombo-embolism-prevention (STEP) QI program with a multidisciplinary team involving hematology, anesthesia, surgery, pharmacy, nursing, and hospital stakeholders/executives. The STEP assessed both surgical risks (low/intermediate/high/major abdominopelvic) and medical risks (age, BMI, heart failure, platelet count, hyperfibrinogenemia, estrogen therapy, active cancer, preoperative immobility, history of VTE, pregnancy) for developing VTE, and they created a comprehensive algorithm which encompassed specific recommendations and helped frontline providers decide VTE prophylaxis. Implementing the algorithm significantly increased their VTE prophylaxis rate from 69 to $91 \%$. Furthermore, they found a statistically significant reduction of postoperative bleeding complications from 10.0 to 6.3 in 1,000 (relative risk reduction $37 \%, p=0.02$ ). They implemented multiple interventions, including staff education, mandatory risk assessment, a smartphone application, medication chart modification, nurse involvement, and an e-learning module. While results from a QI project are occasionally difficult to generalize because each institute has different practices, and although 


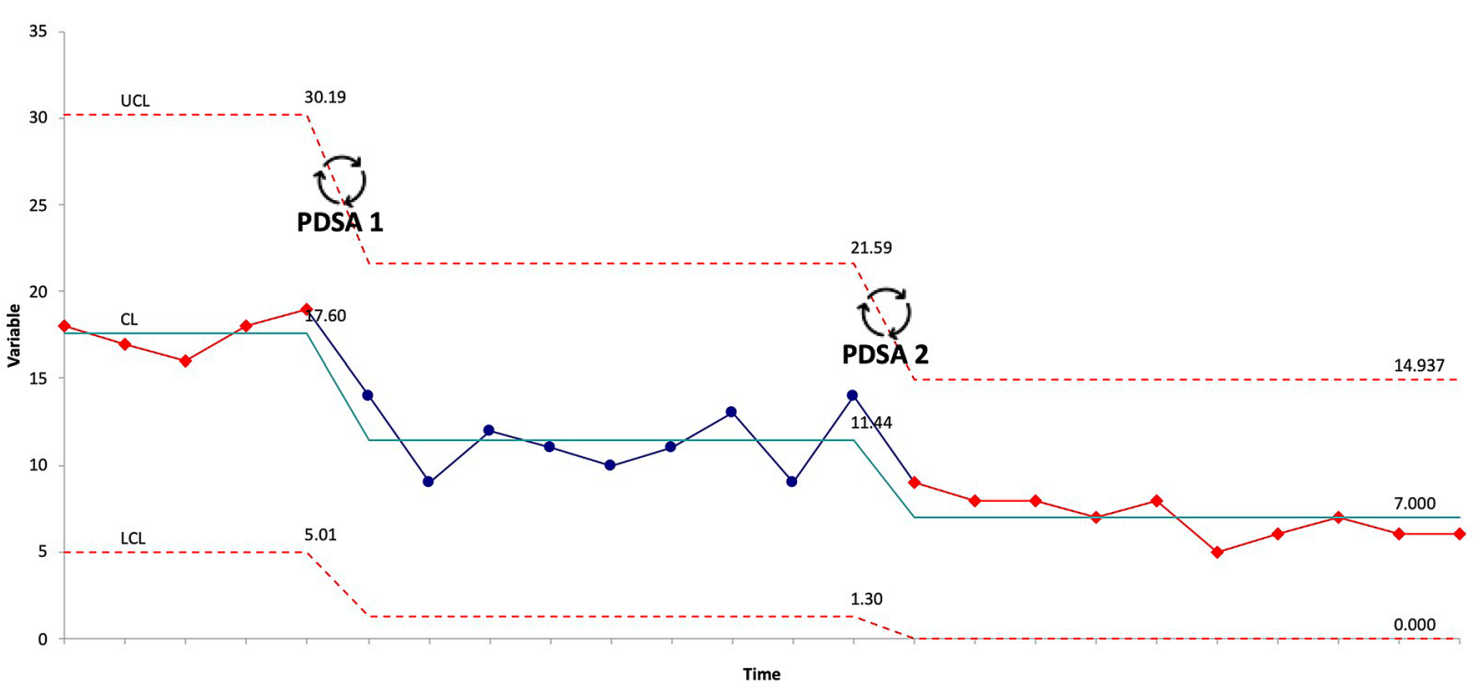

Fig. 2 An example of successful Shewhart control chart. $U C L$ upper control limit, $C L$ center line, $L C L$ lower control limit, PDSA PlanDo-Study-Act. The primary purpose of a control chart is to monitor the data stability by visualizing the sequential improvement. UCL and LCL are calculated by the mean \pm 3 standard deviations, CL rep- resents the mean value, and data outside the control limits are considered abnormal. As long as data are normally distributed, there is $0.3 \%$ chance of the outliers occurring. The figure shows significant improvements after running two cycles of PDSA with considerable changes in control limits the external validity of this QI project needs to be assessed, the magnitude of this specific QI project's achievement is a valuable lesson for clinical quality improvers (CQI) who conduct and drive QI projects in other institutes.

\section{Clinical quality improver: a novel academic career}

The traditional academic pathway is built on scientific publications detailing research done by clinician-scientists, but in the last 2 decades this pathway is becoming more open to clinician-educators as well. Expanding promotion criteria beyond traditional scholarship enables CQIs to also have a career in academia [10]. Currently, academic medical centers in the UK and North America have been striving to improve care quality with strong leadership by CQIs [11]. The realm of QI projects and associated publications in academic journals is growing this field scientifically. The Standard for Quality Improvement Reporting Excellence (SQUIRE) guidelines provide a standard for reporting on QI projects [12], analogous to the CONSORT guidelines for randomized controlled trials [13]. The SQUIRE guidelines provide a framework to report QI projects in healthcare, thus bringing an academic and scholarly lens to these projects. Authors must report a problem description, available knowledge, the rationale behind the interventions, and specific aims following the guidelines, allowing other CQIs to understand clearly why the QI project was implemented. A search of the PubMed database for publications with the term "quality improvement" in their title or abstract returns 407 publications in 2000 compared to 5,687 publications by the end of 2020 (PubMed accessed on December 29, 2020).

\section{How and where to learn QI}

Although QI is gaining popularity in healthcare, in regions where in-person training courses are not available, there appears to be less QI being learned and undertaken. Several key textbooks help one to systematically learn QI, such as The Improvement Guide: A Practical Approach to Enhancing Organizational Performance [14] and The Health Care Data Guide: Learning from Data for Improvement [15]. Moreover, high-quality online materials are provided by credible organizations. For example, the Institute for Healthcare Improvement, located in Boston, has been a leading organization in QI and provides educational materials through its Open School, a series of comprehensive courses to learn the fundamentals of QI and implement a QI project in a local setting with mentorship support [16]. BMJ Quality \& Safety provides various review articles for QI, specifically aiming at how to educate QI in junior faculty members [17], how to learn QI from publications [7], and how to become a champion leader in QI [18]. To deepen QI knowledge, online Master's level courses are also available from several universities, such as Johns Hopkins [19], which have a range of tuition costs. Like in other clinical practice areas, one needs formal education, including didactic lectures, and practical training with mentors, and opportunities to address 
clinical issues. Our hope is to see QI continue to be used by physicians to contribute to improving healthcare systems and resulting in improved patient outcomes.

Acknowledgements As a recipient of Research Commendation, 20212023 Merit Awards Competition, Department of Anesthesiology \& Pain Medicine, University of Toronto, KA acknowledges Department of Anesthesiology \& Pain Medicine, University of Toronto, and Department of Anesthesia and Pain Medicine, Hospital for Sick Children, for their efforts to secure academic time to conduct the current work.

Author contributions YM and KA conceived this paper, wrote the first draft of the manuscript, subsequent iterations of the manuscript and approved the final manuscript.

Funding Not applicable.

\section{Compliance with ethical standards}

Conflict of interest The authors declare that they have no competing interests related to this publication.

\section{References}

1. Kawaguchi M, Tanaka Y, Furuya H. Improving the safety and quality of surgical patient care: what can we learn from quality management of industries? J Anesth. 2015;29:485-6.

2. Kawaguchi M, Ida M, Naito Y. The role of Perioperative Surgical Home on health and longevity in society: importance of the surgical prehabilitation program. J Anesth. 2017;31:319-24.

3. Hines K, Mouchtouris N, Knightly JJ, Harrop J. A brief history of quality improvement in health care and spinal surgery. Glob Spine J. 2020;10:5S-9S.

4. Institute for Healthcare Improvement. http://www.ihi.org/resou rces/Pages/HowtoImprove/ScienceofImprovementEstablishi ngMeasures.aspx. Accessed 11 Dec 2020

5. McGeorge E, Coughlan C, Fawcett M, Klaber RE. Quality improvement education for medical students: a near-peer pilot study. BMC Med Educ. 2020;20:128.

6. Asch SM, Kerr EA, Keesey J, Adams JL, Setodji CM, Malik S, McGlynn EA. Who Is at greatest risk for receiving poor-quality health care? N Engl J Med. 2006;354:1147-56.

7. Glasziou P, Ogrinc G, Goodman S. Can evidence-based medicine and clinical quality improvement learn from each other? BMJ Qual Saf. 2011;20:113-7.
8. Varkey P, Reller MK, Resar RK. Basics of Quality Improvement in Health Care. Mayo Clin Proc. 2007;82:735-9.

9. Chahal R, Alexander M, Yee K, Jun CMK, Dagher JG, Ismail H, Riedel B, Burbury K. Impact of a risk-stratified thromboprophylaxis protocol on the incidence of postoperative venous thromboembolism and bleeding. Anaesthesia. 2020;75:1028-38.

10. Shojania KG, Levinson W. Clinicians in quality improvement. JAMA. 2009;301:766.

11. Staiger TO, Mills LM, Wong BM, Levinson W, Bremner WJ, Schleyer AM. Recognizing quality improvement and patient safety activities in academic promotion in departments of medicine: innovative language in promotion criteria. Am J Med. 2016;129:540-6.

12. Goodman D, Ogrinc G, Davies L, Baker GR, Barnsteiner J, Foster TC, Gali K, Hilden J, Horwitz L, Kaplan HC, Leis J. Explanation and elaboration of the SQUIRE (Standards for Quality Improvement Reporting Excellence) Guidelines, V.2.0: examples of SQUIRE elements in the healthcare improvement literature. BMJ Qual Saf. 2016;25:e7.

13. Schulz KF, Altman DG, Moher D; CONSORT Group. CONSORT. statement: updated guidelines for reporting parallel group randomised trials. BMJ. 2010;2010(340):c332. https://doi. org/10.1136/bmj.c332.

14. Langley GJ, Moen RD, Nolan KM, Nolan TW, Nolan CL, Provost LP. The improvement guide: a practical approach to enhancing organizational performance. Wiley; 2009.

15. Provost LP, Murray SK. The Health Care Data Guide: Learning from Data for Improvement. New York: Wiley; 2011.

16. Institute for Healthcare Improvement. http://www.ihi.org/educa tion/IHIOpenSchool/Pages/default.aspx. Accessed 13 Oct 2020.

17. Myers JS, Wong BM. Measuring outcomes in quality improvement education: success is in the eye of the beholder. BMJ Qual Saf. 2019;28:345-8.

18. Kaminski GM, Britto MT, Schoettker PJ, Farber SL, Muething S, Kotagal UR. Developing capable quality improvement leaders. BMJ Qual Saf. 2012;21:903-11.

19. Johns Hopkins Master course. https://www.jhsph.edu/academics/ online-programs-and-learning/online-programs-for-applied-learn ing/master-of-applied-science-patient-safety-and-healthcare-quali ty/. Accessed 12 Dec 2020

Publisher's Note Springer Nature remains neutral with regard to jurisdictional claims in published maps and institutional affiliations. 